\title{
Qualidade Web para um Prontuário Eletrônico do Paciente
}

\author{
Lourdes Mattos Brasil ${ }^{1}$, Rejane Maria da Costa Figueiredo ${ }^{1}$, Isabelle Domingues Ulysséa ${ }^{2}$; Andréia \\ Patrícia da Silva ${ }^{2}$ \\ ${ }^{1}$ Universidade Católica de Brasília (UCB), Mestrado em Gestão do Conhecimento e da Tecnologia da \\ Informação, SGAN 916 - Módulo B, Campos II, Cep: 70.790 - 160 \\ Brasília, Brasil

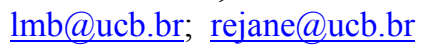 \\ ${ }^{2}$ Universidade Católica de Brasília (UCB), MBA em Gestão de Sistemas de Informação, SGAN 916 - \\ Módulo B, Campos II, cep: 70.790-160 \\ Brasília, Brasil \\ isabelle.ulyssea@terra.com.br; andreiaps@yahoo.com.br
}

\begin{abstract}
Resumo
Este trabalho realiza, a partir das normas de qualidade para software, de requisitos para padrões de software médico e da constante preocupação com a usabilidade, a elaboração de um conjunto de diretrizes de qualidade $W e b$ para um Prontuário Eletrônico do Paciente. A metodologia utilizada foi descritiva. Como resultado, obteve-se uma árvore de requisitos de qualidade para interface de aplicações $W e b$ para Prontuário Eletrônico do Paciente.

Palavras-chave: Sistemas de Informação, Prontuário Eletrônico, Qualidade $W e b$.
\end{abstract}

\begin{abstract}
This work presents, based on software quality standard, medical software requirement standards and the constant concern about usability, the elaboration of a set of web interface quality guidelines for a Medical Electronic Record. The methodology applied was descriptive. As a result, it was achieved a web quality interface requirement tree for a Medical Electronic Record according to software product quality and web software product quality standards.

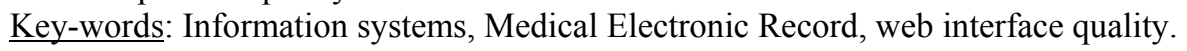

\section{Introdução}

Este modelo tem a finalidade de ser uma base para a Com a ampla e crescente utilização da tecnologia da informação na área de saúde, a qualidade dos serviços de assistência médica tem obtido um considerável ganho de eficiência, precisão e agilidade na realização de seus processos. Tão grande e crescente quanto o uso da tecnologia da informação são as exigências de qualidade dos produtos de software atualmente nessa área [9].

Os vendedores de produtos de software freqüentemente presumem que o fato de possuírem em seu quadro de funcionários especialistas na área de domínio, garante a eficiência do seu produto em ambiente de produção [6]. No entanto, apenas o foco na área de domínio não é suficiente para garantir a qualidade da aplicação. Esforços conjuntos das áreas de tecnologia da informação e saúde em diversos órgãos vêm buscando constantemente estabelecer e homologar um conjunto de diretrizes e normas para estabelecimento de padrões de qualidade de software na área médica.

O Comitê de Trabalho ISO 215, que tem como objetivo estabelecer um padrão internacional para $o$ registro de saúde, o Conselho Federal de Medicina (CFM), o Banco de Dados do Sistema Único de Saúde (DATASUS) e a Sociedade Brasileira de Informática na 
Saúde (SBIS), estão entre os principais órgãos envolvidos nesse processo.

Com a utilização da tecnologia da informação, os registros de saúde dos pacientes - outrora efetuados em papel - estão sendo gradualmente substituídos por sistemas automatizados denominados Prontuários Eletrônicos do Paciente (PEP). Essa nova tecnologia tem a promessa de não apenas substituir o prontuário em papel, mas também elevar a qualidade da assistência à saúde através de novos recursos e aplicações. Com a evolução dos equipamentos de imagens e sinais, cada vez mais documentos em diferentes tipos de mídia podem ser agregados ao prontuário [3]. Considerando-se as facilidades da internet através das tecnologias Web, justifica-se o desenvolvimento desse produto nesse ambiente, pelas facilidades de conectividade, uso, flexibilidade e segurança.

Jacob Nielsen [6] alerta para os riscos a que o paciente está submetido devido a problemas de usabilidade na interface, mencionando o caso de seis pacientes que sofreram queimaduras severas em máquinas de tratamento radioativo devido à falhas na operação do maquinário, cujo painel de controle era de operação complexa e suscetível a erros. Enfatiza ainda os erros de medicação muito comuns em sistemas de prontuários hospitalares, também causados por interfaces com usabilidade problemática, que, como observado em pesquisa de campo publicada no Journal of the American Medical Association (apud [6]), induz o usuário ao erro sem que o mesmo sequer perceba essa condição.

A fim de caracterizar a qualidade $W e b$, esse trabalho definiu diretrizes a serem aplicadas durante o desenvolvimento do projeto de prontuários eletrônicos, de modo a garantir a facilidade da utilização, a concordância com os requisitos do registro de saúde e a usabilidade da aplicação de um sistema web.

\section{Qualidade de Software Produto Web}

Alguns atributos estão relacionados à qualidade da aplicação Web. O conceito sobre o que é uma boa aplicação Web varia em diversos níveis, pois depende do ponto de vista de cada indivíduo. Alguns usuários baseiam seu julgamento na quantidade de conteúdo apresentado, outros na apresentação gráfica e fácil navegação. Ainda pode ser considerado como item relevante ao julgar uma aplicação $W e b$ os serviços oferecidos pelo site.
Uma forma de se garantir a qualidade de um sistema para $\mathrm{Web}$ é poder avaliar a qualidade do produto total, mas para isso é preciso antes definir quais atributos são importantes para tal avaliação, pois cada sistema possui suas necessidades assim como um público definido.

Olsina et al. [7] apresentou um trabalho no qual foi definido um conjunto de atributos e características que permitiram avaliar sites acadêmicos. Para definir tais diretrizes, Olsina et al. [7] elaborou uma "árvore de requisitos de qualidade" a qual descreve um conjunto de atributos que levam à alta qualidade das aplicações $W e b$. $\mathrm{O}$ trabalho teve como base as características de qualidade definidas para software e que são: usabilidade, funcionalidade, confiabilidade, eficiência, portabilidade e manutenibilidade. Como o trabalho considerou o ponto de vista apenas do usuário em geral (estudantes, acadêmicos, professores e patrocinadores em potencial), as características de manutenibilidade e portabilidade (importam mais aos desenvolvedores e gerentes do que aos usuários) foram desconsideradas.

Na Figura 1 apresenta-se um resumo da árvore de requisitos de qualidade de Olsina et al. [7].

\subsection{Usabilidade}

A internet continua evoluindo e passou a invadir cada vez mais a intimidade de empresas, lares, escolas, universidades e muitos outros locais. Atualmente computadores ligados à internet são largamente utilizados.

Uma revolução desse porte, que tem em sua essência a informação, tem alterado fortemente o nosso estilo de vida. Novos conhecimentos, novas tecnologias são criadas e postas à disposição de quem delas precisa. A informação já existente é continuamente trabalhada e aperfeiçoada por pessoas espalhadas por todo o mundo, 24 horas por dia, 7 dias por semana mudando o modo como pensamos, trabalhamos e vivemos.

$\mathrm{Na}$ Era da Informação, é fundamental que todo ser humano possa ter acesso eficaz às redes de informação, em especial à internet. Algumas iniciativas estão sendo desenvolvidas neste sentido, mas para que os esforços online sejam bem sucedidos é fundamental que os websites sejam efetivos, eficientes e satisfatórios para o seu público. Isto pode ser conseguido colocando o humano no centro da tecnologia e não ao contrário; focando a usabilidade desde o começo do processo de design. Pode parecer fácil desenvolver um website, mas fazer um site eficiente e profissional, que realmente atenda ao usuário, é uma tarefa complexa. 


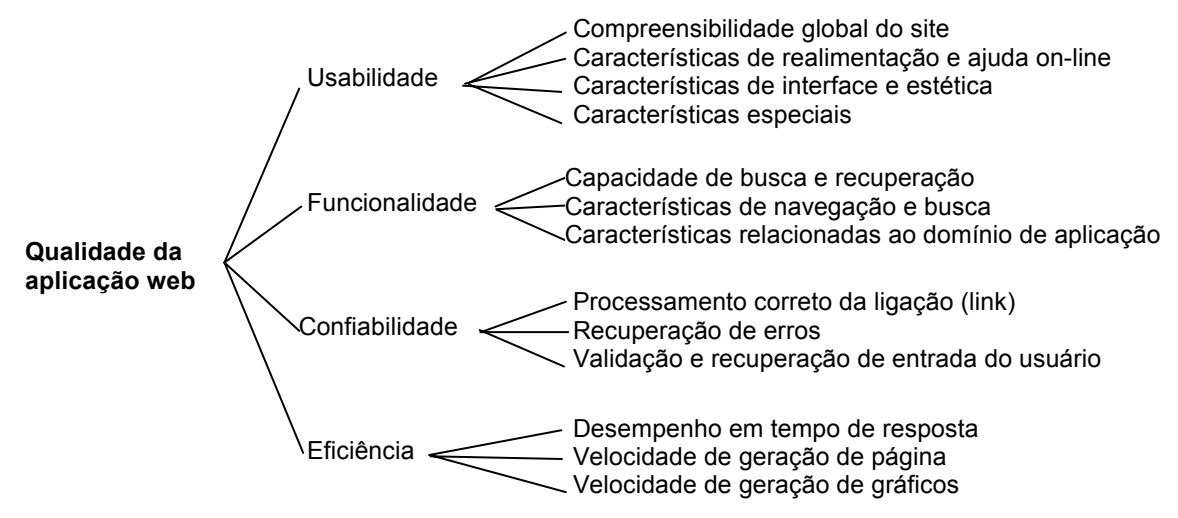

Figura 1 - Resumo da árvore de requisitos de qualidade, adaptado de [8]

\subsubsection{Definição de Usabilidade}

A ISO 9241-11 [2] definiu usabilidade da seguinte forma: "Usabilidade é a eficiência, eficácia e satisfação com a qual os públicos do produto alcançam objetivos em um determinado ambiente".

É preciso, portanto, identificar os objetivos, e decompor a usabilidade (eficácia, eficiência e satisfação) em atributos passíveis de serem verificados e mensurados, assim como o contexto de uso (usuário, tarefa, equipamento e ambiente), conforme estrutura apresentada na Figura 2.
Contexto de uso: usuários, tarefas, equipamento (hardware, software e materiais), e o ambiente físico e social no qual um produto é usado.

Produto: parte do equipamento (hardware, software e materiais) para o qual a usabilidade é especificada ou avaliada.

Objetivo: resultado pretendido.

Eficácia: acurácia e completude com as quais usuários alcançam objetivos específicos.

Eficiência: recursos gastos em relação à acurácia e abrangência com as quais usuários atingem objetivos.

Satisfação: ausência do desconforto e presença de atitudes positivas para com o uso de um produto.

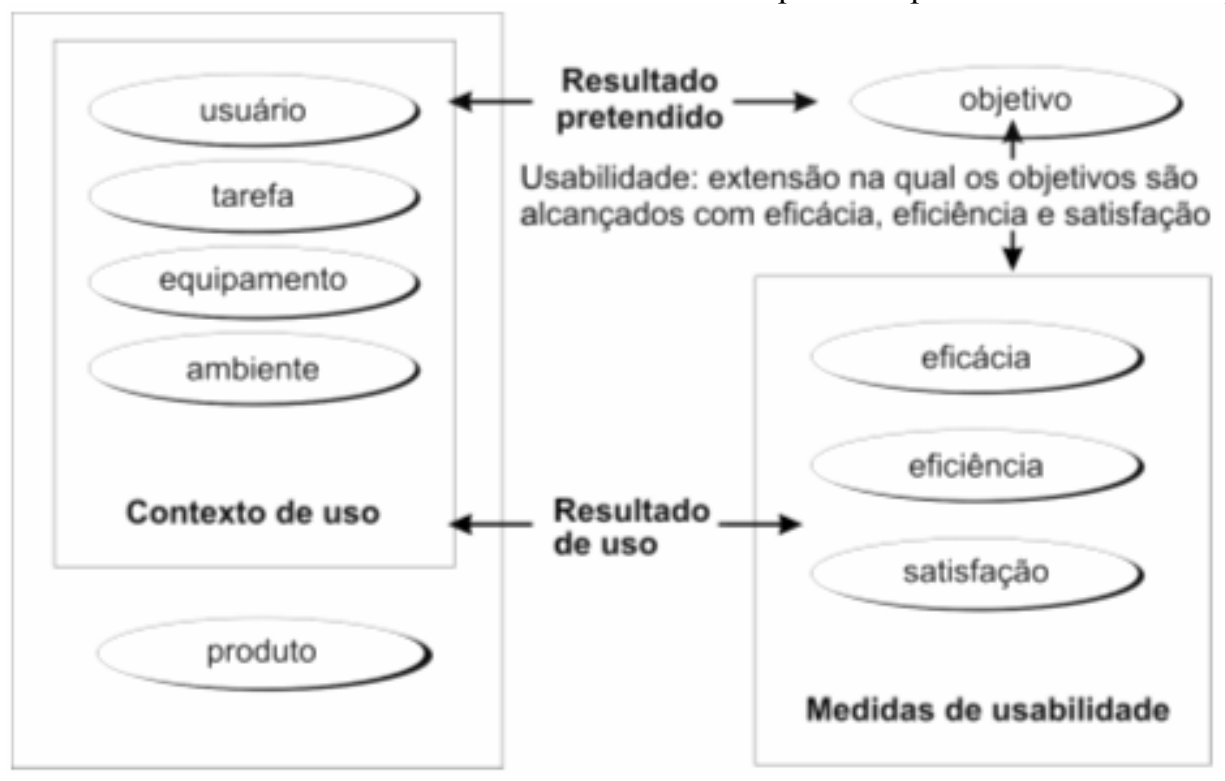

Figura 2 - Resumo da árvore de requisitos de qualidade, adaptado de [8]

Jakob Nielsen [6] descreve cinco atributos da usabilidade: facilidade de aprendizado, eficiência de uso, facilidade de memorização, baixa taxa de erros e satisfação subjetiva. Estes atributos podem ser facilmente 
relacionados aos atributos da ISO 9241-11, mas há outros atributos que devem ser considerados [2]:

Consistência - tarefas similares requerem seqüências de ações similares, assim como ações iguais devem acarretar efeitos iguais. Usar terminologia, leiaute gráfico, conjuntos de cores e de fontes padronizados também são medidas de consistência.

Flexibilidade - refere-se à variedade de formas com que o usuário e o sistema trocam informações. Este atributo diz respeito à capacidade do sistema em se adaptar ao contexto e às necessidades e preferências do usuário, tornando seu uso mais eficiente. Em função da diversidade de tipos de usuários de um sistema interativo, é necessário que sua interface seja flexível o bastante para realizar a mesma tarefa de diferentes maneiras, de acordo com o contexto e com as características de cada tipo de usuário.

Os conceitos de desempenho e satisfação, aliados à facilidade de instalação, manutenção a aprendizado tornaram-se, na década de 90, elementos críticos de sucesso de um produto no mercado mundial [4]. Além de atender melhor as expectativas do mercado existem outras razões para adotar usabilidade desde cedo em um projeto, são elas: redução de erros e de procedimentos de correção; redução do tempo de operação da interface; redução de custos de treinamento, de manutenção e de suporte ao usuário e, como conseqüência, o aumento da eficiência e da efetividade da interface para o seu público. Porém, a necessidade de se preocupar com a estruturação e a apresentação de informações em ambientes digitais, sobretudo em websites, é relativamente recente e crescente na mesma proporção em que cresce também o volume de informações na internet.

A internet introduziu uma complexidade nova para a usabilidade nunca vista antes no software tradicional. Para as organizações, as conseqüências de uma interface gráfica pobre em usabilidade são muito piores quando esta é implementada na internet do que quando é implementada em software tradicional [6].

Por ser um novo meio de comunicação, a internet tem uma linguagem própria construída sobre um espaço hipermídia complexo, com características particulares de acesso remoto a dados, rápida absorção de novidades tecnológicas, publicação dinâmica de informações, velocidades de conexão variáveis, interfaces gráficas mutantes, entre outras. Apesar da internet ter popularizado a usabilidade, acrescentou-lhe novos e grandes desafios.

Dessa forma, são poucos os websites que se enquadram nas características de uma arquitetura adequada ao público potencial, sendo a grande maioria deles criados sem muita preocupação para com o seu usuário, o seu conteúdo e o seu produto. Segundo Jakob Nielsen [6], os usuários não permanecem num site mais do que um minuto se eles não conseguirem navegar e localizar as informações que buscam.

\section{Prontuário Médico}

Prontuário Médico é um conjunto de documentos e informações referentes a um paciente e sua doença, contendo [11]: Identificação individual, social; Origem do mal; Diagnósticos provisório, definitivo e outros; Tratamento; Evolução; Relatórios e Resultado final.

$\mathrm{O}$ prontuário não deve ser visto apenas como o histórico médico resumido do paciente, mas todo acervo documental padronizado, organizado e conciso, referente ao registro dos cuidados prestados ao paciente, assim como aos documentos pertinentes a essa assistência. Trata-se de um documentário de grande valor, elaborado por diversos profissionais de inúmeras áreas. $\mathrm{O}$ prontuário passou a ser visto não apenas como uma peça burocrática para fins da contabilização da cobrança dos procedimentos ou das despesas hospitalares, porém como prova nas possíveis complicações de ordem técnica, ética ou jurídica que possam eventualmente ocorrer.

$\mathrm{Na}$ Resolução CFM n ${ }^{\circ}$ 1.638/2002, Prontuário Médico é definido como:

- Art. $1^{\circ}$ - Definir prontuário médico como o documento único constituído de um conjunto de informações, sinais e imagens registradas, geradas a partir de fatos, acontecimentos e situações sobre a saúde do paciente e a assistência a ele prestada, de caráter legal, sigiloso e científico, que possibilita a comunicação entre membros da equipe multiprofissional e a continuidade da assistência prestada ao indivíduo.

Van Ginneken e Moorman [12] destacam as finalidades de um prontuário:

a) Suporte à assistência ao paciente: como fonte para avaliação e tomada de decisão e como fonte de informação a ser compartilhada entre os profissionais de saúde;

b) Um documento legal dos atos médicos;

c) Suporte à pesquisa: pesquisa clínica, estudos epidemiológicos, avaliação da qualidade do atendimento e ensaios clínicos;

d) Apoio ao ensino para os profissionais de saúde;

e) Gerenciamento e serviços: faturamento, autorização de procedimentos, administração, custos, etc.

\subsection{Prontuário Eletrônico do Paciente}

O prontuário em papel vem sendo utilizado há milhares de anos, e vem passando por diversas transformações ao longo do tempo, principalmente no último século quando se tornou mais sistematizado. Com a evolução da informática nos hospitais e clínicas, surgiu a necessidade de melhorar a eficiência e organização do armazenamento 
das informações de saúde. A solução encontrada foi o desenvolvimento de PEP.

O PEP surgiu não só para substituir o prontuário em papel, mas também para elevar a qualidade da assistência à saúde através de novos recursos e aplicações [9].

Existem várias definições de PEP e, dentre as quais, destacam-se [3]:

Institute of Medicine: "o registro computadorizado de paciente é um registro eletrônico que reside em um sistema especificamente projetado para dar apoio aos usuários, a partir da disponibilidade de dados completos e corretos, lembretes e alertas aos médicos, sistemas de apoio à decisão, links para bases de conhecimento médico, e outros auxílios".

Computer-based Patient Record Institute: "Um registro computadorizado de paciente é uma informação mantida eletronicamente sobre o status e cuidados de saúde de um indivíduo durante toda a sua vida".

\subsubsection{Requisitos para um Sistema de Prontuário Eletrônico do Paciente}

Para um sistema ser considerado um PEP, deve atender aos seguintes requisitos, conforme Quadro 1:

São várias as vantagens de um prontuário eletrônico em relação ao prontuário baseado em papel, que vão desde questões ligadas a maior facilidade de acesso e segurança até oferta de novos recursos, os quais são impossíveis de existir no prontuário em papel, tais como: apoio à decisão, troca eletrônica dos dados entre instituições, etc.

Mas muitas vezes a falta de informação e de confiança, e as dificuldades de acesso de alguns usuários aos meios eletrônicos tornam essas vantagens uma simples ilusão.

É importante destacar que, ao contrário do que se imagina, o PEP é muito mais seguro e tem maior possibilidade de manter a confidencialidade do que os prontuários em papel, no qual o risco de um acesso não autorizado ocorrer é maior que num sistema eletrônico.

É possível também, através do PEP, obter-se uma melhoria na qualidade da assistência à saúde do paciente, melhor gerenciamento dos recursos, melhoria de processos administrativos e financeiros e, ainda, a possibilidade para avaliação da qualidade [12].
Quadro 1 - Características e subcaracterísticas de qualidade de software (adaptado de [5])

\begin{tabular}{|c|c|}
\hline \multicolumn{2}{|r|}{ Conteúdo do Registro } \\
\hline & $\begin{array}{l}\text { Dados uniformes } \\
\text { Formatos e sistemas de codificação padronizados } \\
\text { Dicionário comum de dados } \\
\text { Informações sobre resultados do atendimento e status } \\
\text { funcional }\end{array}$ \\
\hline \multicolumn{2}{|c|}{ Inteligência } \\
\hline & $\begin{array}{l}\text { Suporte à decisão } \\
\text { Lembretes aos médicos } \\
\text { Sistemas de alertas personalizáveis }\end{array}$ \\
\hline \multicolumn{2}{|c|}{ Formato do Registro } \\
\hline & $\begin{array}{l}\text { Lista de problemas na página inicial } \\
\text { Capacidade de navegar pelo prontuário } \\
\text { Integrado entre as especialidades e pontos de atendimento }\end{array}$ \\
\hline \multicolumn{2}{|r|}{ Desempenho do Sistema } \\
\hline & $\begin{array}{l}\text { Rapidez na resposta } \\
\text { Acesso } 24 \text { horas por dia } \\
\text { Disponível nos lugares onde é necessário } \\
\text { Fácil entrada de dado }\end{array}$ \\
\hline \multicolumn{2}{|c|}{ Integração } \\
\hline & $\begin{array}{l}\text { Integrado com outros sistemas de informação (e.g., } \\
\text { radiologia, laboratório, etc.) } \\
\text { Transferência de informação entre as especialidades e } \\
\text { sistemas } \\
\text { Links para literatura científica } \\
\text { Integração com outras instituições } \\
\text { Link para os prontuários dos familiares }\end{array}$ \\
\hline \multicolumn{2}{|c|}{ Relatórios } \\
\hline & $\begin{array}{l}\text { "Documentos Derivativos" (ex: formulários de seguradoras, } \\
\text { etc) } \\
\text { Formatos e interface facilmente personalizáveis } \\
\text { Relatórios clínicos padrões (sumário de alta, etc) } \\
\text { Relatórios personalizáveis para fins específicos } \\
\text { Gráficos }\end{array}$ \\
\hline \multicolumn{2}{|r|}{ Controle e Acesso } \\
\hline & $\begin{array}{l}\text { Fácil acesso para pacientes } \\
\text { Mecanismos para preservar a confidencialidade }\end{array}$ \\
\hline \multicolumn{2}{|r|}{ Treinamento e implementação } \\
\hline & $\begin{array}{l}\text { Necessidade mínima de treinamento para os usuários } \\
\text { utilizarem o sistema } \\
\text { Possibilidade de implantação gradual }\end{array}$ \\
\hline
\end{tabular}




\subsubsection{Necessidade: Padronização de um PEP}

Segundo Van Bemmel (apud [12]) são vários os fatores que definem a necessidade de padronização de um PEP:

a) A diversidade de fontes e termos;

b) A diversidade de plataformas de hardware e software em que os sistemas se encontram;

c) A facilitação da busca de informações;

d) A facilitação da comunicação de informações;

e) Estatística, epidemiologia, prestação de contas, indexação de documentos e pesquisa clínica;

f) A necessidade de integração entre os sistemas;

g) A viabilização de sistemas de apoio à decisão.

h) Levando-se em consideração a necessidade de integração do sistema de PEP com outros sistemas e equipamentos biomédicos, é fundamental a questão dos padrões. Em [3] é citada a elaboração de padrões, que compreende três focos principais de atenção, isto é, vocabulário, conteúdo e arquitetura de sistemas.

Foram ainda incorporadas à lista as diretrizes gerais publicadas por diversos autores como Jakob Nielsen [6], Jeffrey Zeldman [15], entre outros (Figura 3).

Optou-se pela aplicação da árvore de requisitos de Olsina et al. [7] para a categorização dessas diretrizes uma vez que sua estrutura segue o modelo da ISO para avaliações. Sendo assim, a estrutura da árvore foi adequada, considerando-se as características de qualidade de software produto [1].

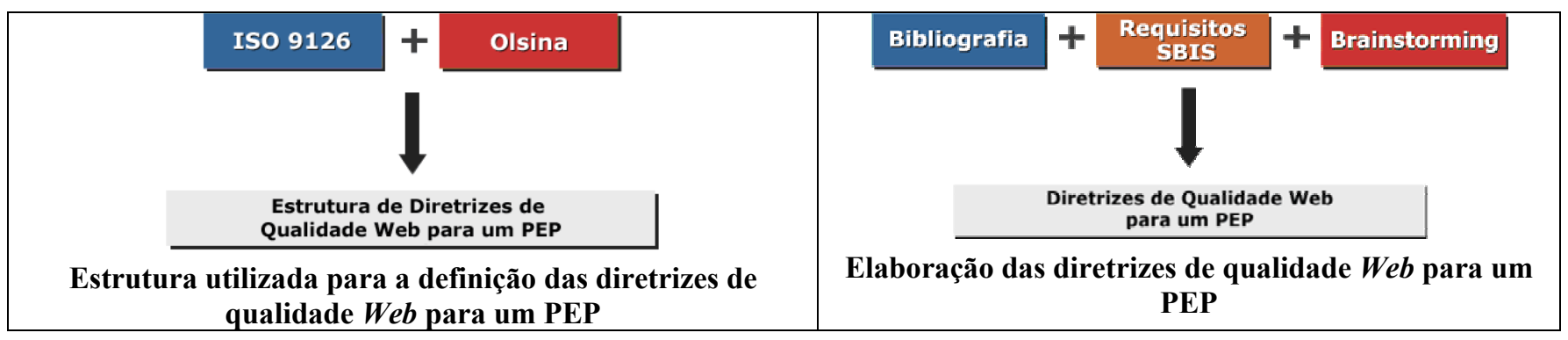

Figura 3 - Processo de Construção das Diretrizes a partir do Referencial Teórico

Foram ainda incorporadas à lista as diretrizes gerais publicadas por diversos autores como Jakob Nielsen [6], Jeffrey Zeldman [15], entre outros.

Optou-se pela aplicação da árvore de requisitos de Olsina et al. [7] para a categorização dessas diretrizes uma vez que sua estrutura segue o modelo da ISO para avaliações. Sendo assim, a estrutura da árvore foi adequada, considerando-se as características de qualidade de software produto [1] (Figura 4).

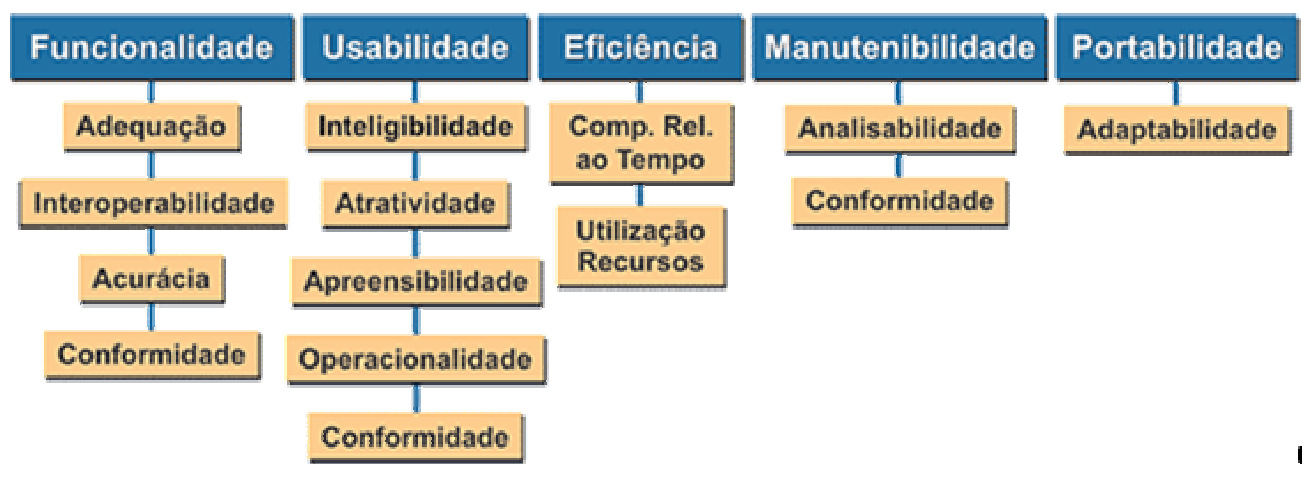

Figura 4 - Árvore das diretrizes de qualidade para um PEP 


\section{Diretrizes de qualidade Web de um PEP}

Em um primeiro momento foram levantadas todas as diretrizes de qualidade em um processo de brainstorming aliada à pesquisa na bibliografia. Elaborou-se a categorização da árvore e, em seguida, as diretrizes foram distribuídas nas categorias pertinentes

\section{Quadro 2 - Exemplo: Diretrizes de Qualidade de}

Interface de um PEP

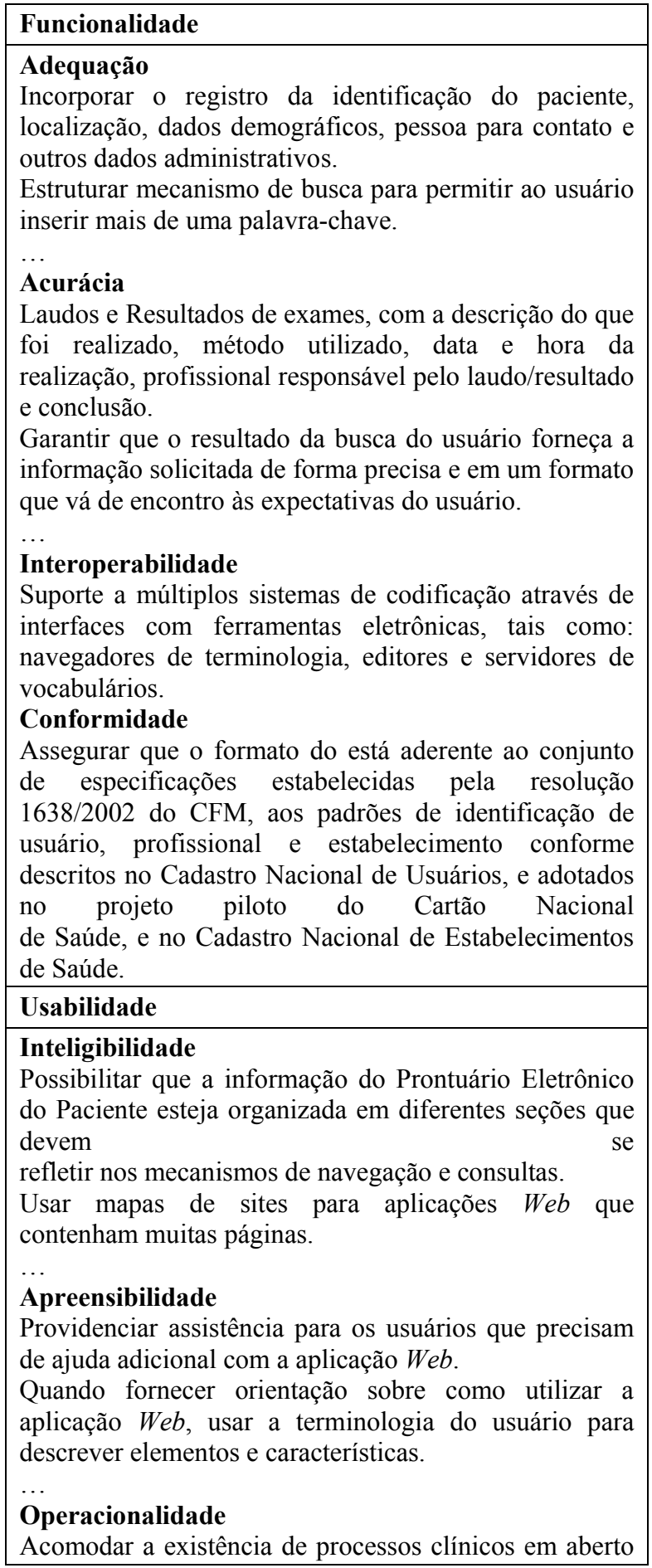

de acordo com sua natureza. A característica de confiabilidade mencionada na ISO 9126-1 [1] foi desconsiderada por ter sido julgada mais relacionada aos motores e ao hardware da aplicação (Figura 4).

No Quadro 2 são exemplificadas as diretrizes de qualidade de interface para um PEP em função da árvore descrita na Figura 4.

(solicitação de exames nunca realizados).

Apresentar dados e informações em um formato que não necessita da conversão pelo usuário. Ex: Utilizar unidade métricas de acordo com os padrões locais.

$\cdots$

Conformidade

Garantir que o formato de itens comuns esteja de forma consistente de uma página para outra. O formato convencional escolhido deve ser familiar para os usuários (ex:. números de telefones devem ter pontuação, ou separação de horas por dois pontos).

\section{Eficiência}

Comportamento em relação ao tempo

Minimizar o tempo requerido para carregar as páginas da aplicação $W e b$.

Avisar aos usuários o tempo de processamento de uma atividade.

...

\section{Utilização de recursos}

Indicar aos usuários o tamanho e o tempo requerido para baixar uma imagem e uma determinada velocidade de conexão.

\section{Manutenibilidade}

Analisabilidade

Utilizar comentários no código para facilitar a manutenção e alterações das páginas.

$\cdots$

\section{Conformidade}

Definir estilo de marcação no código e permanecer nele, criando-se um padrão para as endentações, facilitando assim a leitura e entendimento do código.

Padronizar a interface utilizando folhas de estilo em cascata (CSS) para todos os elementos da aplicação Web.

\section{Portabilidade}

\section{Adaptabilidade}

Projetar, desenvolver e testar para os navegadores e sistemas operacionais mais utilizados.

\section{Conclusão}

O PEP para $W e b$ possui, devido a sua natureza, um grau de exigência maior no que se refere à qualidade da sua interface, pois erros de execução e desempenho podem ocasionar danos irreparáveis para aqueles que dependem do uso desses sistemas.

A união do modelo da árvore de requisitos de qualidade de websites de Olsina et al. [7] a uma norma de qualidade de software possibilita a realização da 
elaboração dos requisitos de qualidade de uma forma completa, tanto em relação ao ambiente - Web - quanto em relação à natureza: software.

A definição das diretrizes de qualidade para um produto sem considerar os requisitos funcionais da própria aplicação é generalista, sendo necessário customizá-las ao produto objeto. Sugere-se, fortemente, a aplicação das diretrizes aqui propostas no desenvolvimento dos produtos desse tipo.

\section{Referências}

[1] $\quad$ ISO/DIS 9241-11: Ergonomic requirements for office work with visual display terminal: guidance usability. Genebra: ISO, 1997.

[2] _. ISO/IEC 9126-1: Software engineering Product quality - Part 1: Quality model. Genebra: ISO, 2001.

[3] COSTA, C.G.A. Desenvolvimento e Avaliação Tecnológica de um Sistema de Prontuário Eletrônico do Paciente, Baseado nos Paradigmas da World Wide Web e da Engenharia de Software. São Paulo: Makron Books, 2001, p.188.

[4] DIAS, C. Usabilidade na web: criando portais mais acessíveis. Rio de Janeiro: Alta Books, 2003.

[5] DICK, R.S.,, STEEN, E.B. \& DETMER,D.E. The Computer-based Patient Record - An Essential Technology for Health Care. Washington, DC: National Academy Press, 1997.

[6] NIELSEN, J. Medical usability: how to kill patients through bad design. Jakob Nielsen's Alertbox. http://www.useit.com/alertbox/20050411.html (17/jun./2005).

[7] OLSINA, L. et al. Specifying quality characteristics and attributes for websites. $1^{\circ}$ ICSE Workshop on $W e b$ Engineering. ACM. Los Angeles. Maio 1999.

[8] PRESSMAN, R. S. Engenharia de software. São Paulo: Makron Books, 1995, p.1056.

[9] ROCHA, A.R., OLIVEIRA, K.M. \& RABELO JR, A. Qualidade de software médico. Simposio Argentino de Informática y Salud. Buenos Aires. 2001.

[10] SBIS. Manual de Requisitos de segurança, conteúdo e funcionalidades para sistemas de registro eletrônico em saúde (RES). http://www.sbis.org.br/GTCERT_20040219_RT_ V2.1.pdf (15/mar./2007).

[11] UNIFENAS - Universidade José do Rosário Vellano. http://www.unifenas.br/ enfcina/semiologiai.htm (1/jun./2005).

[12] VAN GINNEKEN, A.M. \& MOORMAN, P.W.. The Patient Record. In: VAN BEMMEL, J. H. \& [13] MUSEN, M.A. Handbook of medical informatics. Netherlands: Springer-Verlag. 1997.

[14] W3C. World Wide Web Consortium. http://www.w3.org (10/mar/2007).

[15] ZELDMAN, J. Projetando Web Sites Compativeis. Editora: CAMPUS, 2003, p.456.
Lourdes Mattos Brasil concluiu o doutorado em

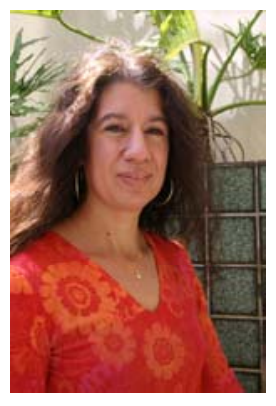

Engenharia Elétrica pela Universidade Federal de Santa Catarina em 1999. De 1997 a 1998 desenvolveu pesquisas em sistemas híbridos pela Université Notre Dame de la Paix, Namur, Belgium. Atualmente é professor adjunto da Universidade Católica de Brasília. Em 2004 coordenou 2 projetos de pesquisa. Atualmente participa de 4 projetos de pesquisa, sendo que coordena 3 destes. Atua na área de engenharia biomédica, com ênfase em informática em saúde. Em suas atividades profissionais interagiu com 276 colaboradores em co-autorias de trabalhos científicos. Em seu currículo Lattes os termos mais freqüentes na contextualização da produção científica, tecnológica e artístico-cultural são: informática em saúde, redes neurais artificiais, artificial intelligence, inteligência artificial em saúde, inteligência artificial, cardiologia, engenharia biomédica, neural networks, hybrid expert systems, informática medica e gestão do conhecimento.

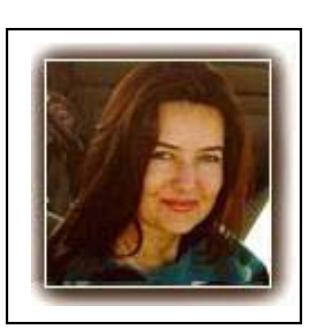

Rejane Maria da Costa Fiqueiredo concluiu o doutorado pela Universidade de São Paulo - USP, com estudos sobre terceirização de serviços de tecnologia da informação. Mestrado pela USP em Ciência da Computação. Atua desde 2002 na Universidade Católica de Brasília, no mestardo MGCTI na área de Gestão da Tecnologia da Informação, e tem orientado dissertações envolvendo sistemas de informação, qualidade de software, escritórios de projetos, acordos de nível de serviços na contratação de serviços de TI, governança em TI, com Cobit e ISO, e trabalhos de gestão ligados aos cursos de Lato Sensu em Engenharia de Software e do MBA Governança em TI. É implementadora MPS$\mathrm{Br}$ e coordenadora do projeto de pesquisa GETIMPS, coordenadora do MBA Governança em TI e do Lato Sensu Engenharia de Software.

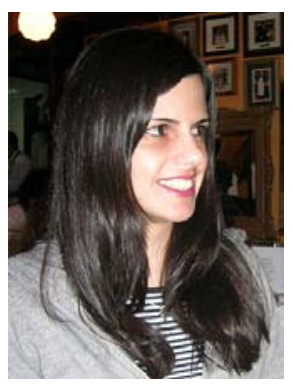

Isabelle Domingues Ulysséa possui graduação em Publicidade e Propaganda pela Universidade de Brasília (1994). Fez o Curso Talento 2002 - Desenvolvedor .NET (2002) pela EFATEC. Em 2005, obteve o título de especialista no MBA em Gestão de Sistemas da Informação na Universidade Católica de Brasília, UCB-DF. Trabalhou como webdesigner na O.M. Comunicação Total e na Interagência Comunicação. 


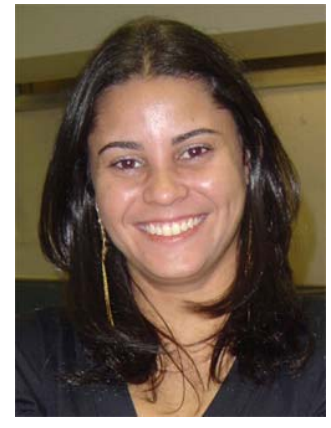

Andréia Patrícia da Silva possui graduação em Processamento de Dados (2000) e MBA em Gestão de Sistemas da Informação (2005) pela Universidade Católica de Brasília, UCBDF. Presta serviço há 6 anos para o Ministério do Esporte como Analista de Sistemas pela empresa SIGMA S/A. Realizou os Cursos: Oracle PL/SQL pelo SENAC; Análise de Requisitos pela EFTI; Flash Básico e Avançado pela CONTRAINING; Vídeo Design Prémiere / After Effects pela USINA e participou do Workshop "Usabilidade de Sites e Portais" promovido pela Terra Fórum. 